\title{
Removable Edges in Near-bricks
}

\author{
Xiumei Wang $\|^{1} \quad$ Cheng He $\|^{2} \quad$ Yixun $\operatorname{Lin}^{1}$ \\ ${ }^{1}$ School of Mathematics and Statistics, Zhengzhou University, Zhengzhou 450001, China \\ ${ }^{2}$ School of Science, Henan University of Technology, Zhengzhou 450001, China \\ received $7^{\text {th }}$ October 2011, revised $5^{\text {th }}$ December 2012, accepted $10^{\text {th }}$ July 2013.
}

For a brick apart from a few small graphs, Lovász (1987) proposed a conjecture on the existence of an edge whose deletion results in a graph with only one brick in its tight cut decomposition. Carvalho, Lucchesi, and Murty (2002) confirmed this conjecture by showing the existence of such two edges. This paper generalizes the result obtained by Carvalho et al. to the case of irreducible near-brick, where a graph is irreducible if it contains no induced odd path of length 3 or more. Meanwhile, a lower bound on the number of removable edges of matching-covered bipartite graphs is presented.

Keywords: perfect matching, removable edge, brick, near-brick

\section{Introduction}

The notion of brick plays an important role in matching theory. A graph is matching-covered if it is connected and each of its edges is contained in a perfect matching. A graph $G$ is bicritical if $G-\{u, v\}$ has a perfect matching for any two distinct vertices $u, v$ in $G$. A brick is a 3-connected and bicritical graph. Clearly, a brick is matching-covered and non-bipartite. Four important matching-covered graphs, which are bricks, are shown in Figure 1.

Let $G$ be a matching-covered graph. The "tight cut decomposition" of $G$, which consists of bricks and braces, was introduced in [4] and [6]. Here, a brace is a matching-covered bipartite graph $H$ with bipartition $(A, B)$ such that for any distinct vertices $u_{1}, u_{2} \in A$ and $v_{1}, v_{2} \in B$, the graph $H-\left\{u_{1}, u_{2}, v_{1}, v_{2}\right\}$ has a perfect matching. For a proper nonempty set $S$ of $V(G)$, let $\delta(S)$ denote the set of edges of $G$ with exactly one end in $S$. If $S=\{v\}$, we simply write $\delta(v)$ instead of $\delta(\{v\})$. For a cut $\delta(S)$ of $G$ with two shores $S$ and $\bar{S}$, where $\bar{S}=V(G) \backslash S, \delta(S)$ is trivial if either $|S|=1$ or $|\bar{S}|=1$, and is tight if $|\delta(S) \cap M|=1$ for every perfect matching $M$ of $G$. The crucial point of the tight cut decomposition is the following result (Theorem 37.8 of [8] or Lemma 1.4 of [6]): A matching-covered graph has no nontrivial tight cuts if and only if it is either a brick or a brace.

\footnotetext{
${ }^{\dagger}$ Email: wangxiumeiezzu.edu.cn. Supported by NSFC (grant no. 11101383 and 11201432).

$\ddagger$ Supported by NSFC (grant no. 11201121). 


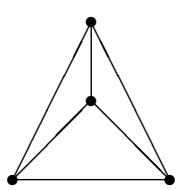

$K_{4}$

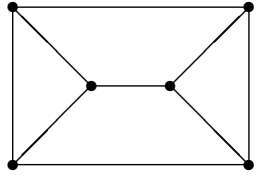

$\bar{C}_{6}$

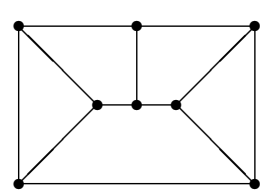

$R_{8}$

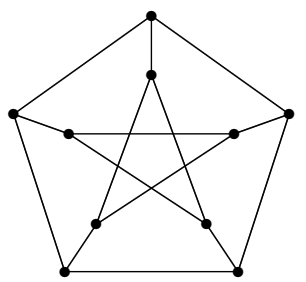

The Petersen graph

Figure 1. Four important matching-covered graphs

For a cut $C$ of $G$ with $C=\delta(S)$, the graphs $G / S$ and $G / \bar{S}$ which are obtained from $G$ by contracting $S$ and $\bar{S}$ to single vertices, respectively, are called $C$-contractions of $G$. The two contracted vertices are denoted by $s$ and $\bar{s}$, respectively. If $C$ is a tight cut of $G$, then both $G / S$ and $G / \bar{S}$ are matching-covered.

A tight cut decomposition procedure of $G$ can be stated as follows (see [6]). Let $C$ be a nontrivial tight cut of $G$. Let $G_{1}$ and $G_{2}$ be the two $C$-contractions of $G$, which are matching-covered. If either $G_{1}$ or $G_{2}$ has a nontrivial tight cut, then we can carry out the $C$-contractions of that graph and obtain smaller matching-covered graphs. This process can be repeated until each resulting graph is either a brick or a brace. The set of all bricks and braces produced, which are uniquely determined by $G$ (see Lemma 2.1), is a tight cut decomposition of $G$. For convenience, we refer to a brick or brace in a tight cut decomposition of $G$ as a brick or brace of $G$. Note that any brick or brace of $G$ has at least four vertices.

Let $b(G)$ denote the number of the bricks in a tight cut decomposition of $G$. An edge $e$ of $G$ is removable if $G-e$ is matching-covered, and is b-invariant if it is removable and $b(G-e)=b(G)$. Edmonds, Lovász, and Pulleyblank [4] proved that the dimension of the perfect matching polytope of $G$ is $|E(G)|-|V(G)|+1-b(G)$ (see Theorem 7.6.6 of [7]). Lovász [6] proved that the dimension of the matching lattice of $G$ is $|E(G)|-|V(G)|+2-b(G)$. In [6], Lovász gave a characterization of matching lattice. The hard part of this characterization is the case when $b(G)=1$ and the underlying simple graph of the only brick of $G$ is different from the Petersen graph. Motivated by this, Lovász proposed the following conjecture: Every brick different from $K_{4}, \bar{C}_{6}$, and the Petersen graph has a $b$-invariant edge. Carvalho, Lucchesi, and Murty confirmed this conjecture by proving an even stronger version as follows.

Theorem 1.1 [2] Every brick different from $K_{4}, \bar{C}_{6}, R_{8}$, and the Petersen graph has two b-invariant edges.

A near-brick is a matching-covered graph $H$ with $b(H)=1$. Clearly, a brick is a near-brick. If a brick $H$ has a $b$-invariant edge $e$, then $H-e$ is a near-brick. An induced path of a graph is a path in which all internal vertices have degree 2 in this graph. A graph is irreducible if it contains no induced odd path of length 3 or more. Here, a path is odd or even according to the parity of its length. A brick is irreducible. This is because a brick is 3 -connected and so has no vertices of degree 2 . However, a near-brick is not necessarily irreducible. For example, subdividing an edge of a graph in Figure 1 by two vertices results in a near-brick, which is not irreducible. The main result of this paper is the following theorem which is an extension of Theorem 1.1 to irreducible near-bricks.

Theorem 1.2 [2] Let $G$ be a near-brick which is irreducible and is different from $K_{4}, \bar{C}_{6}, R_{8}$, and the Petersen graph. Then $G$ has two b-invariant edges. 
The paper is organized as follows. In Section 2, we present some results on matching-covered graphs which are used in this paper. In Section 3, we give a lower bound on the number of removable edges for matching-covered bipartite graphs, which is used in Section 4 Section 4 is devoted to the proof of the main result.

\section{Preliminaries}

The following result obtained by Lovász is an important property of tight cut decompositions.

Lemma 2.1 [6] Any two tight cut decompositions of a matching-covered graph yield the same list of bricks and braces (except possibly for multiplicities of edges).

Lemma 2.1 implies that the numbers of bricks, braces, and braces with four vertices in a tight cut decomposition of a matching-covered graph $G$ are invariants of $G$. From Lemma 2.1, we also see that a graph $G$ is a near-brick if and only if for every tight cut $C$ of $G$, one of the $C$-contractions is bipartite and the other one is also a near-brick.

For a set $X \subseteq V(G)$, let $N(X)$ denote the set of vertices of $G$ which is not in $X$ but has neighbors in $X$, and write $N[X]=N(X) \cup X$. For convenience, we simply write $N(v)$ and $N[v]$ instead of $N(\{v\})$ and $N[\{v\}]$, respectively. A graph $G$ is elementary if the set of edges each of which lies in a perfect matching of $G$ induces a connected subgraph of $G$. The following lemma says that in the case of bipartite graphs "elementary" is an alternate definition of "matching-covered".

Lemma 2.2 (Theorem 4.1.1 in [7]) Let $G$ be a bipartite graph with bipartition $(A, B)$. Then the following are equivalent:

(i) $G$ is elementary;

(ii) $|A|=|B|$ and for every nonempty proper subset $X$ of $A,|N(X)| \geq|X|+1$;

(iii) $G$ is matching-covered.

For a matching-covered graph $G$, a $b$-invariant edge of $G$ is removable, but the converse is not true. In fact, if $e$ is a removable edge of $G$, then $b(G-e) \geq b(G)$ (see Theorem 2.17 in [2]). We now give a characterization of $b$-invariant edges.

Lemma 2.3 Let $\delta(S)$ be a tight cut of a matching-covered graph $G$ and e an edge of $G$. Then $e$ is $b$-invariant in $G$ if and only if $e$ is b-invariant in each of $G / S$ and $G / \bar{S}$ which contains it.

Proof: Write $C=\delta(S)$. Suppose first that $e$ is a $b$-invariant edge in $G$. Then $G-e$ is matching-covered and $b(G-e)=b(G)$. Assume, without loss of generality, that $e$ is an edge of $G / S$. Let $e^{\prime}$ be any edge of $G / S-e$. Then $G-e$ has a perfect matching $M$ containing $e^{\prime}$, which is also a perfect matching of $G$. Recall that $C$ is a tight cut of $G$. So $|M \cap C|=1$, and thus $M \cap E(G / S-e)$ is a perfect matching of $G / S-e$ containing $e^{\prime}$. This implies that $G / S-e$ is matching-covered, that is, $e$ is removable in $G / S$. By symmetry, if $e$ is an edge of $G / \bar{S}$, it is also removable in $G / \bar{S}$.

Note that $C-e$ is a tight cut of $G-e$, and $(G-e) / S$ and $(G-e) / \bar{S}$ are two $(C-e)$-contractions of $G-e$. Combining these observations with the definition of a tight cut decomposition and the fact that $(G-e) / S=G / S-e$ and $(G-e) / \bar{S}=G / \bar{S}-e$, we have

$$
b(G / S)+b(G / \bar{S})=b(G)=b(G-e)=b(G / S-e)+b(G / \bar{S}-e) \geq b(G / S)+b(G / \bar{S}) .
$$


This implies that $b(G / S-e)=b(G / S)$ and $b(G / \bar{S}-e)=b(G / \bar{S})$, and so $e$ is $b$-invariant in each of $G / S$ and $G / \bar{S}$ which contains it.

Conversely, suppose that $e$ is $b$-invariant in each of $G / S$ and $G / \bar{S}$ which contains it. Then $b(G / S-e)=$ $b(G / S), b(G / \bar{S}-e)=b(G / \bar{S})$, and $G / S-e$ and $G / \bar{S}-e$ are both matching-covered. Let $f$ be an arbitrary edge of $G-e$. Suppose, without loss of generality, that $f \in E(G / S-e)$. Then $G / S-e$ has a perfect matching $M_{1}$ containing $f$, and $G / \bar{S}-e$ has a perfect matching $M_{2}$ containing the edge in $M_{1}$ incident with the contracted vertex $s$ of $G / S$ (it is possible that this edge is $f$ ). Then $M_{1} \cup M_{2}$ is a perfect matching of $G-e$ containing $f$. Therefore $G-e$ is matching-covered, and so $e$ is removable in $G$. Since

$$
b(G-e)=b(G / S-e)+b(G / \bar{S}-e)=b(G / S)+b(G / \bar{S})=b(G),
$$

$e$ is $b$-invariant in $G$. The proof is complete.

The matching-covered graphs have the following basic property: If $G$ is a graph other than $K_{2}$ and $G^{\prime}$ results from $G$ by subdividing an edge with two vertices, then $G$ is matching-covered if and only if $G^{\prime}$ is matching-covered (see Remark (1) in p.145 of [7]). To say conversely, let $P$ be an induced odd path of $G$. Then $G$ is matching-covered if and only if the graph $G_{P}$ obtained from $G$ by replacing $P$ by a single edge, say $e_{P}$, is matching-covered. A path of $G$ is removable if it is an induced odd path of $G$ and the graph obtained from $G$ by deleting all edges and internal vertices of this path is matching-covered. Clearly, $P$ is removable in $G$ if and only if $e_{p}$ is removable in $G_{P}$. If a matching-covered graph $G$ has an induced odd path of length at least three, then each edge in this path is not removable and so is not $b$-invariant. In this paper we mainly investigate matching-covered graphs which are irreducible.

\section{Bipartite graphs}

Recall that a brace is a matching-covered bipartite graph free of nontrivial tight cuts. A brace with four vertices is a 4-cycle with possible multiple edges. Each vertex in a brace on 6 or more vertices has at least three neighbors.

Lemma 3.1 [3] In a brace on 6 or more vertices, every edge is removable.

Lemma 3.2 Let $G$ be a matching-covered bipartite graph, and $\delta(S)$ a nontrivial cut of $G$. Then $\delta(S)$ is a tight cut of $G$ if and only if there is a subset $Z$ of one color class of $G$ with $|N(Z)|=|Z|+1$ and $S=N[Z]$.

Proof: Write $C=\delta(S)$ and let $(X, Y)$ be a bipartition of $G$. Then $|X|=|Y|$ since $G$ has perfect matchings. The proof of the "if " part is easy.

To show the "only if" part, suppose that $C$ is a tight cut of $G$. Let $Z$ be the smaller one of $S \cap X$ and $S \cap Y$, say $Z=S \cap X$. Since every perfect matching of $G$ has exactly one edge in $C,|S \cap Y|=|Z|+1$ and then $Z$ is a proper subset of $X$. Since $C$ is nontrivial, $|S| \geq 3$, and so $|Z| \geq 1$. By Lemma 2.2. $|N(Z)| \geq|Z|+1=|S \cap Y|$. We further assert that $N(Z) \subseteq S \cap Y$. This is because if there is an edge $e$ joining a vertex in $Z$ and a vertex in $N(Z)$ but not in $S \cap Y$, then a perfect matching of $G$ containing $e$ has at least three edges in $C$, one is $e$ and the other two connect vertices in $X \backslash Z$ and $S \cap Y$, a contradiction. Therefore $N(Z)=S \cap Y$, and thus $|N(Z)|=|Z|+1$ and $S=N[Z]$. The proof is complete.

As it turns out, if $v$ is a vertex of a matching-covered graph $H$ with exactly two neighbors in $H$ (it is possible that there are multiple edges incident with $v$ and so $d_{H}(v) \geq 3$ ), then $\delta(N[v])$ is a nontrivial 
tight cut of $H$. When $H$ is bipartite, this is a special case of Lemma 3.2 with $Z=\{v\}$. Furthermore, Lemma 3.2 implies that, for a nontrivial tight cut $C$ of a matching-covered bipartite graph $G$, the two $C$-contractions of $G$ are also bipartite.

For a matching-covered graph $G$ which is irreducible, we make a convention on the tight cut decomposition procedure as follows: In the procedure of the decomposition, whenever there is a vertex $v$ of degree 2 in the current graphs, we first use the tight cut of form $\delta(N[v])$. We call such decomposition a simple tight cut decomposition.

Lemma 3.3 Let $G$ be a matching-covered bipartite graph which is irreducible. Then

(i) the number of vertices of degree 2 in $G$ is at most $\frac{|V(G)|}{2}-1$, and

(ii) for each brace with four vertices in a simple tight cut decomposition of $G$, there are at most two edges which are not multiple.

Proof: (i) Let $(X, Y)$ be a bipartition of $G$ and $S$ the set of vertices of degree 2 in $G$. Then $S$ is an independent set. If $S \cap X=X$, then $\bar{S}$ is a subset of $Y$ and so is independent, contradicting $d(v) \geq 3$ for each $v \in \bar{S}$. Thus $S \cap X \subset X$. By symmetry, $S \cap Y \subset Y$. If one of $S \cap X$ and $S \cap Y$ is empty, then the assertion is true since $|X|=|Y|$. So we assume that $S \cap X \neq \emptyset$ and $S \cap Y \neq \emptyset$. By Lemma2.2.

$$
|\bar{S}| \geq|N(S \cap X)|+|N(S \cap Y)| \geq|S \cap X|+|S \cap Y|+2=|S|+2,
$$

which implies (i).

(ii) We show a more general result: When a simple tight cut decomposition procedure is performed, if a graph $H$ is irreducible, then the two $C$-contractions are also irreducible. Assume $C=\delta(S)$. Suppose, to the contrary, that $H / S$ is not irreducible. Then $H / S$ has two adjacent vertices of degree 2, one of which is the contracted vertex $s$ (since $H$ is irreducible) and the other has degree 2 in $H$. By the convention of the simple tight cut decomposition procedure, the shore $S$ is in the form of $\delta(N[x])$, where $x$ is a vertex of $H$ with degree 2. Since $|\delta(S)|=2$, the two neighbors of $x$ also have degree 2 in $H$, a contradiction to the fact that $H$ is irreducible. The proof leads to (ii).

We now present a lower bound on the number of removable edges for matching-covered bipartite graphs. Let $\beta(G)$ denote the number of braces with four vertices in a tight cut decomposition of a matching-covered graph $G$. Let $\mathcal{G}$ be a simple tight cut decomposition of $G$. Recall that $\beta(G)$ is an invariant of $G$, which implies that $\beta(G)$ is also the number of braces with four vertices in $\mathcal{G}$. Because each graph in $\mathcal{G}$ has at least four vertices, we have $|\mathcal{G}| \leq \frac{|V(G)|}{2}-1$ by induction on the number of nontrivial tight cuts used in obtaining $\mathcal{G}$, and so $\beta(G) \leq \frac{|V(G)|}{2}-1$.

Theorem 3.4 Let $G$ be an irreducible matching-covered bipartite graph other than $K_{2}$. Then $G$ has at least $|E(G)|-2 \beta(G)$ removable edges.

Proof: By Lemma 2.3 and Lemma 3.1, the non-removable edges of $G$ are distributed within the braces with four vertices in a simple tight cut decomposition of $G$. Thus the assertion follows from Lemma 3.3 (ii).

Let us make several remarks concerning with Theorem 3.4 .

(1) The graph in Figure 2 shows that the lower bound in Theorem 3.4 is sharp. The eight edges in this graph which are incident with vertices of degree 2 are non-removable and the remaining eight edges are removable. 


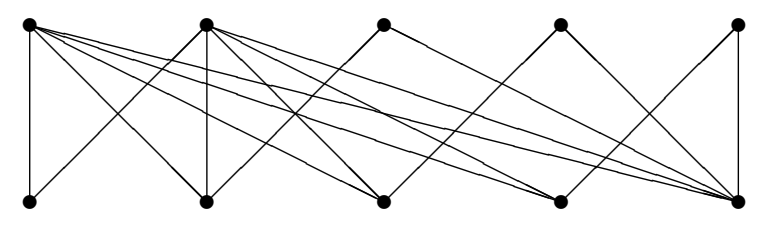

Figure 2. A matching-covered graph with $|E(G)|-2 \beta(G)$ removable edges

(2) Using Theorem 3.4 and the fact $\beta(G) \leq \frac{|V(G)|}{2}-1$, we see that an irreducible matching-covered bipartite graph $G$ other than $K_{2}$ has at least $|E(G)|-|V(G)|+2$ removable edges. From this we can deduce the following two known results. A graph is a minimal elementary graph if it is elementary but deleting any edge from it results in a non-elementary graph.

Corollary 3.5 [7] Let $G$ be a minimal elementary bipartite graph other than $K_{2}$. Then the number of edges of $G$ whose ends have degree 2 is at least $|E(G)|-|V(G)|+2$.

Proof: By Lemma 2.2 a bipartite graph is elementary if and only if it is matching-covered. Combining this with the assumption that $G$ is a minimal elementary graph, we see that $G$ has no removable edges, and hence is not irreducible by Theorem 3.4. For each maximal induced odd path of $G$ with length 3 or more, which is not included in any other induced odd path of $G$, replace it by an edge. Then the resulting graph $G^{\prime}$ is an irreducible matching-covered bipartite graph, and so has at least $\left|E\left(G^{\prime}\right)\right|-\left|V\left(G^{\prime}\right)\right|+2$ removable edges. Noting that $\left|E\left(G^{\prime}\right)\right|-\left|V\left(G^{\prime}\right)\right|=|E(G)|-|V(G)|$, we see that $G$ has at least $|E(G)|-|V(G)|+2$ removable induced odd paths with length 3 or more, which implies that $G$ has at least $|E(G)|-|V(G)|+2$ edges whose ends have degree 2 in $G$.

Corollary 3.6 [5] Let $G$ be an elementary bipartite graph which is neither a cycle nor a $K_{2}$. Then the number of reducible ears (removable induced odd paths) of $G$ is at least $|E(G)|-|V(G)|+2$.

Proof: From the proof of Corollary 3.5, we see that the assertion is true.

\section{Proof of the maim result}

In this section we prove Theorem 1.2

Let $G$ be a near-brick which is irreducible and is different from $K_{4}, \bar{C}_{6}, R_{8}$, and the Petersen graph. To prove that $G$ has two $b$-invariant edges, we need only show the case when $G$ is simple since multiple edges are $b$-invariant. If $G$ is a brick, the assertion follows from Theorem 1.1 Suppose that $G$ is not a brick. Let $G^{\prime}$ be the brick in a tight cut decomposition of $G$. Since a brick is 3-connected, the minimum degree of $G^{\prime}$ is at least three. We distinguish two cases according to whether $G^{\prime}$ has contracted vertices of degree 3 or not.

Case 1: The brick $G^{\prime}$ has a contracted vertex $v$ of degree 3. Then the edges incident with $v$ form a tight cut of $G$, say $C$. Let $\bar{S}$ be the shore of $C$ such that $\bar{s}=v$ is the contracted vertex of $G^{\prime}$. Then $G / S$ is a matching-covered bipartite graph, $G / \bar{S}$ is a near-brick, and both of them have 4 or more vertices. Write $H_{v}=G / S$. Then $H_{v}$ has only one contracted vertex $s$, and the degree of $s$ in $H_{v}$ is 3 . Let $h$ be the number of removable edges of $H_{v}$ which are not incident with $s$. Using Lemma 2.3 and the fact that for a bipartite graph, any removable edge of it is $b$-invariant, we see that if $h \geq 2$, then $G$ has two $b$-invariant edges. We then show that $h \geq 2$ in the following. 
Because a matching-covered graph is 2-connected, so is $H_{v}$. Then each vertex of $H_{v}$ has at least two neighbors. Recall that the graph $G$ is irreducible and the only contracted vertex $s$ in $H_{v}$ has degree 3 . The graph $H_{v}$ has no vertices of degree 2 adjacent, i.e., $H_{v}$ is irreducible. By Theorem 3.4 .

$$
h \geq\left|E\left(H_{v}\right)\right|-2 \beta\left(H_{v}\right)-3 \geq\left|E\left(H_{v}\right)\right|-\left(\left|V\left(H_{v}\right)\right|-2\right)-3=\left|E\left(H_{v}\right)\right|-\left|V\left(H_{v}\right)\right|-1 .
$$

By Lemma 3.3 (i), the number of vertices of degree 2 in $H_{v}$ is at most $\frac{\left|V\left(H_{v}\right)\right|}{2}-1$. Combining this with the fact that $\left|E\left(H_{v}\right)\right|$ is one half the sum of the degrees of the vertices of $H_{v}$, we have $\left|E\left(H_{v}\right)\right| \geq \frac{5\left|V\left(H_{v}\right)\right|+2}{4}$. If $\left|V\left(H_{v}\right)\right| \geq 8$, then $h \geq\left\lceil\frac{\left|V\left(H_{v}\right)\right|-2}{4}\right\rceil \geq 2$. We now suppose that $\left|V\left(H_{v}\right)\right| \leq 6$.

Let $(X, Y)$ be a bipartition of $H_{v}$ such that $s$ belongs to $Y$. Since $G$ is simple, in $H_{v}$ the edges not incident with $s$ are not multiple. Since $s$ has at least two neighbors in $H_{v}$, there are at most two edges in $H_{v}$ with the same ends one of which is the contracted vertex $s$. If $\left|V\left(H_{v}\right)\right|=4$, then $H_{v}$ has two vertices of degree 2 adjacent, a contradiction. Thus $\left|V\left(H_{v}\right)\right|=6$, and so $\left|E\left(H_{v}\right)\right| \leq 9$ since each vertex in $Y$ has degree at most 3. By inequality (1), $h \geq 2$ holds if $\left|E\left(H_{v}\right)\right|=9$. We may suppose that $\left|E\left(H_{v}\right)\right| \leq 8$. Then $H_{v}$ has a vertex of degree 2 in $Y$, say $u$. Let $u^{\prime}$ be the vertex in $X$ other than the two neighbors of $u$. Then $u^{\prime}$ is adjacent to the two vertices in $Y$ other than $u$. Because $H_{v}$ is irreducible and has at most two multiple edges, the two neighbors of $u$ both have degree 3 or more, and one of them has three neighbors. We claim that there are no multiple edges incident with the neighbors of $u$. If not, since the multiple edges are also incident with $s$, the degree of $s$ in $H_{v}$ is 4 , a contradiction. Therefore $H_{v}$ is simple, and so is $K_{3,3}-u u^{\prime}$. The four edges not incident with $u$ and $u^{\prime}$, two of which are not incident with $s$, are removable in $H_{v}$. It follows that $h \geq 2$.

Case 2: The graph $G^{\prime}$ has no contracted vertex of degree 3. Then $G^{\prime}$ is different from $K_{4}, \bar{C}_{6}, R_{8}$, and the Petersen graph. We proceed by induction on $|E(G)|$. Let $m_{0}$ be the minimum number of edges of a near-brick. If $|E(G)|=m_{0}$, then $G$ is a brick since otherwise there would be a contradiction to the minimality of $m_{0}$. So the assertion is true for this extremal case. Assume below that $|E(G)|>m_{0}$ and the assertion holds for fewer edges.

Let $C$ be an arbitrary nontrivial tight cut in the tight cut decomposition procedure of $G$ which produces $G^{\prime}$. Then $C$ is also a tight cut of $G$. Recall that one of the $C$-contractions of $G$ is a bipartite graph and the other is a near-brick. Among all these nontrivial tight cuts, we may take one $C$ with $C=\delta(S)$ such that $G / S$ is bipartite and $|\bar{S}|$ is as small as possible. Then $G / S$ is a brace, for otherwise $G / S$ can be decomposed further in the tight cut decomposition procedure, contradicting the minimality of $\bar{S}$. Moreover, the near-brick $G / \bar{S}$ is different from $K_{4}, \bar{C}_{6}, R_{8}$, and the Petersen graph. This is because $G^{\prime}$ is the brick of $G / \bar{S}$ which is different from these four graphs.

If the brace $G / S$ has at least six vertices, then each vertex of $G / S$ has at least three neighbors, and so the degree of $s$ in $G / S$, which is equal to the degree of $\bar{s}$ in $G / \bar{S}$, is at least three. Because $G$ is irreducible, so is $G / \bar{S}$. Since $G / \bar{S}$ has fewer edges than $G$, by induction hypothesis, $G / \bar{S}$ has two $b$-invariant edges. By Lemma 3.1. every edge of $G / S$ is removable, and so is $b$-invariant. By Lemma 2.3 the two $b$-invariant edges of $G / S$ are $b$-invariant in $G$.

If $G / S$ has only four vertices, then it is a 4-cycle with multiple edges. By our assumption, $G$ is simple and has no vertices of degree 2 adjacent. So one vertex in $\bar{S}$, say $v_{1}$, has degree 2 in $G$, and the other two vertices, which are neighbors of $v_{1}$, has degree 3 or more. Then $G / S$ is a 4-cycle such that all edges incident with $s$ are multiple. This implies that the degree of $s$ in $G / S$ is at least four, hence so is the degree of $\bar{s}$ in $G / \bar{S}$. Consequently, $G / \bar{S}$ is irreducible, and so has two $b$-invariant edges by induction hypothesis. Note that in $G / S$ each edge incident with $s$ is $b$-invariant. By Lemma 2.3 again, the two $b$-invariant edges 
of $G / \bar{S}$ are $b$-invariant in $G$. This completes the proof of Theorem 1.2

The condition of Theorem 1.2 that the graph is irreducible cannot be omitted: the graphs in Figure 3(a) and 3(b), for instance, are near-bricks but not irreducible, and has at most one $b$-invariant edge. The graph shown in Figure 3(c) is an irreducible near-brick and has two $b$-invariant edges $e_{1}$ and $e_{2}$.

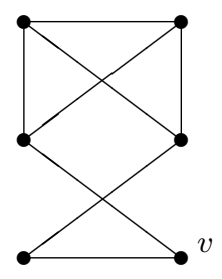

$(a)$

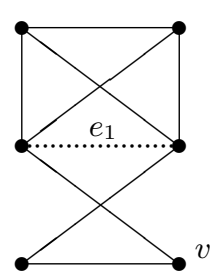

(b)

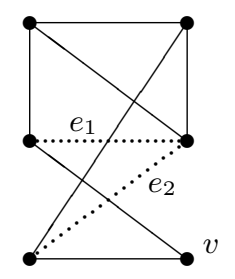

$(c)$

Figure 3. Three near-bricks and their $b$-invariant edges

\section{Acknowledgements}

The authors would like to thank the anonymous referees for their helpful comments on improving the representation of the paper.

\section{References}

[1] J.A. Bondy, U.S.R. Murty, Graph Theory, Springer-Verlag, Berlin, 2008.

[2] M.H. de Carvalho, C.L. Lucchesi, U.S.R. Murty, On a conjecture of Lovász concerning bricks I. The characteristic of a matching-covered graph, J. Combin. Theory, Ser. B, 85 (2002) 94-136.

[3] M.H. de Carvalho, C.L. Lucchesi, U.S.R. Murty, Ear decomposition of matching-covered graphs, Combinatorica, 19 (1999) 151-174.

[4] J. Edmonds, L. Lovász, W.R. Pulleyblank, Brick decompositions and matching rank of graphs, Combinatorica, 2(3)(1982) 247-274.

[5] P. Hansen, F. Zhang, M. Zheng, Perfect matchings and ears in elementary bipartite graphs, Discrete. Math., 176 (1997) 131-138.

[6] L. Lovász, Matching structure and the matching lattice, J. Combin. Theory, Ser. B, 43(1987) 187222.

[7] L. Lovász, M.D. Plummer, Matching Theory, Elsevier Science Publishers, B. V. North Holland, 1986.

[8] A. Schrijver, Combinatorial Optimization: Polyhedra and Efficiency, Springer-Verlag, Berlin 2003. 